Draft Version 1

October 2019

\title{
A Sober Second Thought? A Pre-Registered Experiment on the Effects of Mindfulness Meditation on Political Tolerance
}

Michael Bang Petersen $1 *$ \& Panagiotis Mitkidis2,3

1Department of Political Science, Aarhus University, Aarhus, Denmark

${ }_{2}$ Department of Management, Aarhus University, Aarhus, Denmark

3Social Science Research Institute, Duke University, USA

* Corresponding author: michael@ps.au.dk

\begin{abstract}
Mindfulness meditation is increasingly promoted as a tool to foster more inclusive and tolerant societies and, accordingly, meditation practice has been adopted in a number of public institutions including schools and legislatures. Here, we provide the first empirical test of the effects of mindfulness meditation on political and societal attitudes by examining whether completion in a 15-minute mindfulness meditation increases tolerance towards disliked groups relative to relevant control conditions. Analyses of data from a pilot experiment $(N=54)$ and a pre-registered experiment $(N=171)$ provides no evidence that mindfulness meditation increases political tolerance. Furthermore, exploratory analyses show that individual differences in trait mindfulness is not associated with differences in tolerance. These results suggest that there is reason to pause recommending mindfulness meditation as a way to achieve democratically desirable outcomes or, at least, that short-term meditation is not sufficient to generate these.
\end{abstract}


To show political tolerance is to support the political rights of disliked groups. As Western democracies become increasingly diverse, the question of how to promote political tolerance across ethnic, racial, and political divisions has emerged as a key challenge. However, as summarized in Gibson (2011), "micro-level analysis of change [in political tolerance] is as rare as it is important."

Political tolerance is commonly conceptualized as a "sober second thought", i.e., the reasoned rejection of the initial negative affect towards particular political groups (Gibson, 1998; Marcus et al., 1995). Consequently, previous studies on the promotion of political tolerance have mainly provided people with explicit reasons in favor of tolerance but have generally only found small effects (Gibson, 1998; Petersen et al., 2011). The present study, in contrast, proceeds from the observation that the activation of a "sober second thought" is not just a matter of reasoned motivation but also depends on abilities (see also Halperin et al., 2014). To achieve tolerance, people need to suppress or diffuse initial negative affective reactions towards social or political groups. Doing so requires abilities of self-control or emotionregulation (Halperin et al., 2014). If most past interventions have had limited success, this could reflect that they have not targeted these abilities.

Interventions that target emotion-regulation abilities are receiving increasing attention within the psychological sciences (Gross, 2013). In particular, an emerging view is that training in mindfulness can increase emotion-regulation abilities. Mindfulness or "nonjudgmental attention to experiences in the present moment" (Tang et al., 2015) is typically trained via meditation practices involving "self-regulation of attention" (Bishop et al., 2004). Mindfulness meditation typically involves sustained attention (Smallwood \& Schooler, 2015), (e.g., to breath), attention switching (from thoughts to breath), and the inhibition of elaborated processing (e.g., ruminating - Watkins, 2008). Through mindfulness meditation, one might (i) 
become able to note arising emotions and (ii) resist acting on them, potentially enabling the second sober thought of tolerance.

In line with this, several commentators, journalists, and researchers have highlighted the society-wide adoption of mindfulness as a way to more open and inclusive societies (Wright, 2017), and government-funded programs on mindfulness are currently being rolled out in public institutions across a number of Western countries, including schools and even legislatures (Bristow, 2019). Yet, presently there is no evidence in favor or against the effectiveness of such interventions. No empirical test exists of whether mindfulness meditation is effective in promoting political tolerance or even whether it affects societal views in general.

The aim of the present study is thus to provide the first experimental test of (i) the causal effect of mindfulness meditation on political tolerance and (ii) the presumed mechanism of increased suppression or diffusion of negative emotion. The importance of this aim is buttressed by a recent study suggesting that mindfulness meditation might, in fact, have negative effects on ethnical behavior in interpersonal situations (Schindler et al., 2019).

\section{Method}

To assess the causal effect of mindfulness meditation, we ran two experimental studies. The two studies were almost identical. One served as an initial (i) pilot study and the other is (ii) pre-registered and better powered replication. The pre-registration for the latter study is available at the Open Science Framework: https://osf.io/s2r9t/?view_only=3973a24a96c8494a81d952008f654eae. Any deviation from this pre-registration is either noted here in the main text or in the Online Supplementary Materials (SOM1).

In both studies, each participant was seated in front a computer in a separate room without any other participants. All information was presented to participants on the computer 
screen. After obtaining simple demographic information, we exposed participants to eight political and social groups and asked them which of these groups they liked the least. Specifically, we followed a previous study on political tolerance conducted in Denmark (cite) and listed the following political groups: Muslims, Christian fundamentalists, Islamic fundamentalists, people on the extreme left-wing, people on the extreme right-wing, the Antifa, and neo-Nazis. This way of asking constitutes the foundation for state-of-the-art measures of political tolerance ("the least liked"-approach) as subsequent questions can then be directed towards a group that the individual participant objectively dislikes and, hence, can be an object of tolerance. Subsequently, they answered a series of questions about their emotional reactions to their "least liked"-group. Specifically, respondents were asked to indicate the extent to which they felt "anger", "fear", "disgust", and "contempt" towards the group. Answers were recorded on a 7-point scale from "Not at all" to "Very Strongly". These measures allow us to test whether mindfulness meditation allows political tolerance to be less influenced by initial emotional reactions. In line with the pre-registration, for the pre-registered study, the main text reports the results of a simple, additive scale of negative affect (pilot study: $\alpha=.73$; pre-registered study: $\alpha=.70$ ), recoded to vary between 0 and 1 . In the Online Supplementary Materials, we consider two alternative pre-registered operationalizations of the emotion measures (see SOM2 and SOM3).

After completion of the emotion measures, participants were randomly assigned to either two (in the pilot study) or three (in the pre-registered study) conditions. In the pilot study, participants were either assigned the Mindfulness Meditation Condition or a MindWandering Condition. In the pre-registered study, participants were either assigned to the Mindfulness Meditation Condition, the Mind Wandering Condition or a pure Control Condition. 
In the Mindfulness Meditation Condition, participants were asked to put on headphones and to follow the auditory instructions as closely as possible. They then closed their eyes and listened to a guided 15-minute breathing meditation previously used with positive results in research on the cognitive effects of meditation (Hafenbrack et al., 2014). Our choice to use a short meditation treatment reflects that this type of guided meditation is commonly available in apps and is the type of meditation most likely to be adopted on a wide scale in, for example, public institutions such as schools. Thus, the study provides a test of a minimal but widely available mindfulness intervention. Furthermore, it should be noted that several previous studies have reported that 15 minutes of mindfulness meditation (or even less) is enough to reduce other normatively-undesirable psychological phenomena such as the reliance on cognitive biases (Hafenbrack et al., 2014) and internal cognitive distractions (Weger, Hooper, Meier, \& Hopthrow, 2012) and to enhance concentration (Kuo \& Yeh, 2015) and negotiations performance (Reb \& Narayanan, 2014). Hence, the 15 minutes Mindfulness Meditation Condition is not intended to induce changes in traits related to emotion-regulation but rather to induce a short-term mental state of mindfulness in which increased emotionregulation is attainable.

As our main control condition, we utilized a Mind Wandering Condition (Hafenbrack et al., 2014). The condition was designed to mimic "a waking, baseline mental state" Hafenbrack et al. (2014: 371) and such a baseline has been utilized in a number of prior studies (e.g., Arch \& Craske, 2006). In this condition, participants were also asked to put on headphones and to follow the auditory instructions. Then, they completed a 15-minute task in which they were repeatedly asked "to think of whatever came to mind" Hafenbrack et al. (2014: 371). In the pre-registered study, we also included a pure Control Condition, where participants were simply led to the next part of the study without any interruption. This allows us to assess 
whether any form of distraction between the recall of negative affect towards groups and expressions of political tolerance is enough to increase political tolerance.

After completing any tasks associated with the assigned condition, participants completed the dependent measure of political tolerance. Specifically, we used the following set of questions about the participant's "least liked"-group, taken from prior research in Denmark (Petersen et al., 2011): (1) Representatives of [group] should not be allowed to express themselves in public debate; (2) The police should have better opportunities for tapping telephones owned by [group]; (3) Representatives of [group] should have the right to speak at secondary schools; (4) [group] should be allowed to hold demonstrations. Answers were obtained on 5-point scales ranging from "Completely Agree" to "Completely Disagree". Following the pre-registration, for the pre-registered study, we analyze the dependent variable in two ways. First, we use an additive scale with items 1 and 2 reverse coded (pilot study: $\alpha=$ .79 ; pre-registered study: $\alpha=.76$ ), which is rescaled to vary between 0 and 1 . We analyze this scale using t-tests and ordinary least squares regression with unstandardized regression coefficients reported. Second, we use structural equation modeling to measure political tolerance as a latent variable. For these models, we report standardized coefficients. In the preregistration, we specified directional hypotheses but, as a number of findings go in the opposite direction than specified in these hypotheses, we report two-sided p-values.

Participants for both the pilot study and the pre-registered study were drawn from the subject pool of the local behavioral lab, a diverse subject pool extending beyond students. The studies were approved by the Human Subjects Committee associated with the lab. Fiftyfour individuals (30 males and 24 females, age: $\mathrm{M}=25, \mathrm{SD}=5.1$ ) participated in the pilot study. In the pre-registered study, we aimed for 200 participants. This allows us to detect a directionally-hypothesized and medium-sized effect of mindfulness meditation on political tolerance $(r=.25)$ with power .90 relative to each control condition. In line with the pre- 
registration, we had to terminate the data collection prior to reaching the goal of 200 due to time constraints. Our final sample consisted of 171 participants (83 males and 94 females, age: $\mathrm{M}=24, \mathrm{SD}=3.1)$. Given the directional hypotheses of higher political tolerance in the Mindfulness Meditation Condition, this leaves us with a power of .86 for comparisons between the Mindfulness Meditation Condition and each of the control conditions.
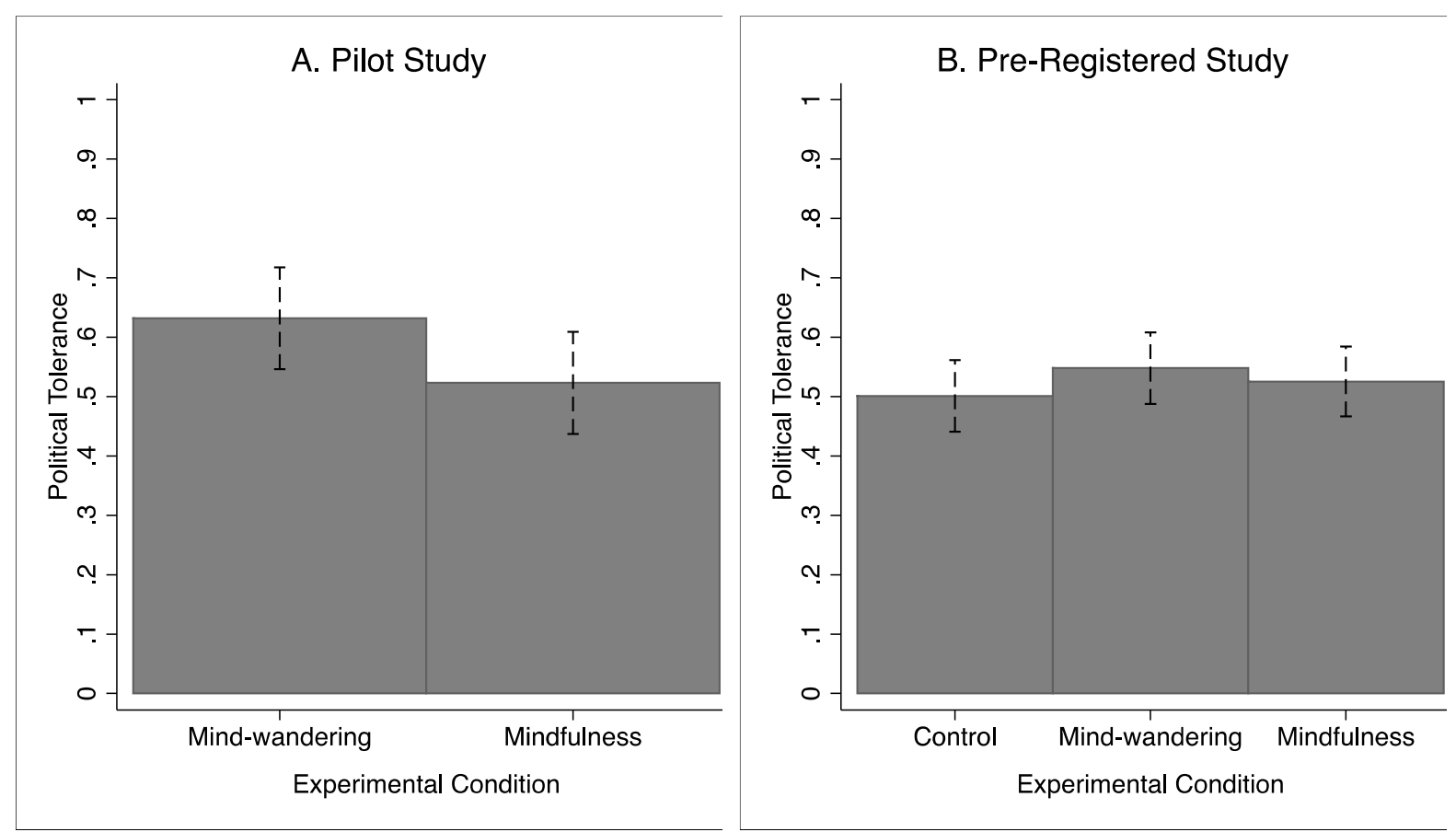

Figure 1. Mean political tolerance on a scale of $0-1$ across conditions in a pilot study $(\mathrm{N}=54)$ and a pre-registered study $(\mathrm{N}=171)$.

\section{Results}

Does a short mindfulness meditation task increase political tolerance? No. Figure 1 displays the mean level of political tolerance across the experimental conditions for both the pilot study (Panel A) and the pre-registered study (Panel B). Against the hypothesized effect, the pilot study showed a marginally significant difference in political tolerance between the conditions with higher tolerance in the Mind-wandering Condition relative to the Mindfulness Condition relative $(\mathrm{M}=.63$ vs. $\mathrm{M}=.52, \mathrm{t}(52)=1.80, \mathrm{p}=.08)$. When the effect of condition on political 
tolerance was estimated using structural equation modeling, the difference reached conventional levels of significance $(\beta=-.28, p=.044)$. In the pre-registered study, with higher power, no evidence of any differences in political tolerance across conditions were observed. Political tolerance was not significantly higher in either the Mind-wandering Condition $(\mathrm{M}=.55$, $\mathrm{t}(113)=.53, \mathrm{p}=.60)$ or the pure Control Condition $(\mathrm{M}=.50, \mathrm{t}(113)=-.59, \mathrm{p}=.56)$ and the Mindfulness Condition $(\mathrm{M}=.52)$. Furthermore, when estimated using structural equation modeling, the effects of both the Mind-wandering Condition $(\beta=.05, \mathrm{p}=.63)$ and the Control Condition $(\beta=-.04, p=.71)$ relative to the Mindfulness Condition continued to be insignificant. It should be noted that in the pre-registered study, as in the pilot study, political tolerance was descriptively higher in the Mind-Wandering Condition relative to the Mindfulness Meditation Condition.

Does a short mindfulness meditation task reduce the effect of negative affective on political tolerance? No. To test this, we regressed political tolerance on the experimental conditions, the self-reported emotions, and the interaction between the two using both ordinary least squares regression and structural equation modeling. The full models are available in the Supplementary Online Materials (SOM2). In the pilot study, these interaction models compared the effects of emotions in the two available conditions: Mindfulness and Mind-wandering. The models revealed an insignificant association between self-reported negative affect and political tolerance in the Mindfulness Condition (OLS: $b=-.05, p=.80$; SEM: $\beta=.03, p=.90$ ). Furthermore, this association was not significantly higher in the Mind-wandering Condition (OLS: $\mathrm{p}=.34$; SEM: $\mathrm{p}=.22$ ) and, hence, there was no conclusive evidence in favor of the hypothesis that mindfulness meditation relative to mind-wandering decreased the reliance on initial affect when forming political tolerance judgments towards disliked groups. In the preregistered study, with higher power, we observed a significant negative effect of negative affect on political tolerance such that more negative affect was associated with higher tolerance (OLS: 
$\mathrm{b}=-.40, \mathrm{p}=.002 ;$ SEM: $\beta=-.38, \mathrm{p}=.004)$. However, again, this association was not significantly more negative in neither the Mind-wandering Condition (OLS: $\mathrm{p}=.94$; SEM: $\mathrm{p}=$ .89) nor the Control Condition (OLS: $p=.11$; SEM: $p=.11$ ). It should be noted that there is some evidence that proceeding more quickly from the affect measure to the tolerance measure - as was the case in the Control Condition - does increase the association between the two measures but, given the lack of difference between the Mind-wandering and Mindfulness Conditions, this reflects more a matter of distraction than a matter of mindfulness. As noted in the pre-registration, there is a risk of bias in these analyses. Given that the affect measure is observed rather than experimentally manipulated, it is possible that the estimated associations are affected by, for example, the specific choice of "least liked"-group, which simultaneously could influence affect and tolerance judgments (see Petersen et al., 2011). In the Online Supplementary Materials, this is corrected for and, in addition, exploratory analyses are presented that correct for a number of potential demographic confounders (SOM4). These additional analyses do not change the above conclusions.

Exploratory analyses of the role of potential moderators and trait mindfulness. Overall, both the pilot study and the pre-registered study did not support that the induction of a mindful mental state increases political tolerance. In this section, we conduct a number of explorative analyses to address different arguments for why we fail to obtain such support. To maximize power, we combine the pilot study and the pre-registered study in these analyses and analyze the combined data using OLS regression. Beyond the difference in control conditions, the two studies collected identical measures. First, we address the potential role of a range of moderators. Thus, it is possible that the positive effects of mindfulness only emerge for some individuals. However, we find no evidence that the differences in expressed tolerance between the Mindfulness Condition relative to Mind-Wandering Condition is conditioned by having no versus any experience with meditation $(\mathrm{p}=.43)$; by being high or low in trait mindfulness 
(measured using the Mindful Attention Awareness scale, Brown \& Ryan, 2003; $\mathrm{p}=.19$ ); by being left- or right-wing $(\mathrm{p}=.96)$ and, hence, having political values or not often argued to be aligned with values of tolerance (Altemeyer, 1988); or by being high or low in need for cognitive closure $(\mathrm{p}=.47)$ and, hence, having a personality aligned with the value of tolerance or not (Roets \& Van Hiel, 2011). Second, it is plausible that the lack of effect reflects that the meditation task is too short to induce true mindfulness. However, we observe that there is no significant correlation between trait mindfulness and political tolerance $(r=-.05, p=.49)$. Even if a prolonged mindfulness intervention did increase trait mindfulness, the present evidence suggests that this might not influence political tolerance.

\section{Discussion and Conclusion}

Confirmatory analyses of the effects of a short mindfulness meditation on political tolerance suggests that a mindful state does not increase political tolerance. Furthermore, exploratory analyses of individual differences in trait mindfulness also suggests that such differences are not reliably associated with differences in political tolerance. These findings question the increasingly common assumption that a wider adoption of mindfulness practices could pave the way for more inclusive, tolerant societies. While the intrapersonal or interpersonal benefits of mindfulness practice are receiving increasing documentation (Eberth \& Sedlmeier, 2012), the present data provide reasons to pause regarding the wider societal benefits of such practice. At the same time, it is important to note the present analyses mainly speak to the effects of shortterm nature of the mindfulness intervention and, hence, studies using more prolonged training could yield different outcomes.

Theoretically, the present findings could reflect that the psychological challenges involved in supporting the granting of political rights to disliked groups should not be conceptualized as a simple exercise in the suppression or diffusion of emotion (although, see 
Halperin et al., 2014). Potentially, the impetus to grant rights in the face of negative of affect could reflect attachments to democratic norms that are themselves affective in nature (Petersen et al., 2011) or countervailing feelings of compassion towards the individual group members. If so, this could imply that other mental exercises would be more efficient in promoting political tolerance. There is an increasing awareness that different meditation exercises produce different outcomes (Singer \& Engert, 2019) and that the mental states of "presence" trained with the most common types of meditation (especially, breathing meditation) might not impact moral behavior (Thupten, 2019). In tandem, these observations suggest that meditation practices that facilitate better perspective-taking abilities (e.g., thought-observation meditation) or more compassion (e.g., loving-kindness meditation) could hold more promise for increasing political tolerance in the increasingly diverse societies of today.

\section{References}

Altemeyer, B. (1988). Enemies offreedom: Understanding right-wing authoritarianism. Jossey-Bass.

Arch, J. J., Craske, M. G. (2006). Mechanisms of mindfulness: Emotion regulation following a focused breathing induction. Behavior Research and Therapy, 44, 1849-1858.

Bishop SR; et al. (2004) Mindfulness: A proposed operational definition. Clinical Psychology: Science and Practice 11(3): 230-41.

Bristow, J. (2019). Mindfulness in politics and public policy Jamie Bristow. Current Opinion in Psychology, 28, 87-91.

Brown, K. W., \& Ryan, R. M. (2003). The benefits of being present: mindfulness and its role in psychological well-being. Journal of personality and social psychology, 84(4), 822.

Eberth, J., \& Sedlmeier, P. (2012). The effects of mindfulness meditation: a metaanalysis. Mindfulness, 3(3), 174-189. 
Gibson, J. L. (1998). A sober second thought: An experiment in persuading Russians to tolerate. American Journal of political science, 42, 819-850.

Gibson, J. L. (2011) Political intolerance in the context of democratic theory. In Godin E. The Oxford Handbook of Political Science (pp. 1-21).

Gross, J. J. (Ed.). (2013). Handbook of emotion regulation. Guilford publications.

Hafenbrack, A. C., Kinias, Z., \& Barsade, S. G. (2014). Debiasing the mind through meditation: Mindfulness and the sunk-cost bias. Psychological science, 25(2), 369-376.

Halperin, E., Pliskin, R., Saguy, T., Liberman, V., \& Gross, J. J. (2014). Emotion regulation and the cultivation of political tolerance: Searching for a new track for intervention. Journal of Conflict Resolution, 58(6), 1110-1138.

Kuo, C. Y., \& Yeh, Y. Y. (2015). Reset a task set after five minutes of mindfulness practice. Consciousness and Cognition, 35, 98-109.

Marcus, G. E., Sullivan, J. L., Theiss-Morse, E., \& Wood, S. L. (1995). With malice toward some: How people make civil liberties judgments. Cambridge University Press.

Petersen, M., Slothuus, R., Stubager, R., \& Togeby, L. (2011). Freedom for all? The strength and limits of political tolerance. British Journal of Political Science, 41(3), 581-597.

Reb, J., \& Narayanan, J. (2014). The influence of mindful attention on value claiming in distributive negotiations: Evidence from four laboratory experiments. Mindfulness, 5(6), 756-766.

Roets, A., \& Van Hiel, A. (2011). Allport's prejudiced personality today: Need for closure as the motivated cognitive basis of prejudice. Current Directions in Psychological Science, 20(6), 349-354.

Schindler, S., Pfattheicher, S., \& Reinhard, M. A. (2019). Potential negative consequences of mindfulness in the moral domain. European Journal of Social Psychology. 
Singer, T., \& Engert, V. (2019). It matters what you practice: Differential training effects on subjective experience, behavior, brain and body in the ReSource Project. Current Opinion in Psychology, 28, 151-158.

Smallwood, J., \& Schooler, J. W. 2015. The science of mind wandering: Empirically navigating the stream of consciousness. Annual Review of Psychology, 66, 487-518.

Tang YY, Holzel BK, \& Posner MI (2015) The neuroscience of mindfulness meditation. Nature Reviews Neuroscience 16(4): 213-25.

Thupten, J. (2019). The question of mindfulness' connection with ethics and compassion. Current Opinion in Psychology, 28, 71-75.

Watkins, E. R. 2008. Constructive and unconstructive repetitive thought. Psychological Bulletin, 134, 163-206.

Weger, U. W., Hooper, N., Meier, B. P., \& Hopthrow, T. (2012). Mindful maths: Reducing the impact of stereotype threat through a mindfulness exercise. Consciousness and Cognition, 21(1), 471-475.

Wright, R. (2017). Why Buddhism is true: The science and philosophy of meditation and enlightenment. Simon and Schuster. 